\title{
Long distance contributions to the rare kaon decay
}

$K \rightarrow \pi \ell^{+} \ell^{-}$

\section{Norman Christ ${ }^{a}, \mathrm{Xu} \mathrm{Feng}^{a}$, Andreas Jüttner $^{b}$, Andrew Lawson $^{* b}$, Antonin Portelli ${ }^{b}$, Christopher Sachrajda ${ }^{b}$}

${ }^{a}$ Physics Department, Columbia University, New York NY 10027, USA

${ }^{b}$ School of Physics and Astronomy, University of Southampton Southampton, SO17 1BJ, UK

Email: nhc@phys.columbia.edu, pkufengxu@gmail.com,

juettner@soton.ac.uk, allg13@soton.ac.uk, antonin.portelli@me.com,

ctsesoton.ac.uk

The rare decays of a kaon into a pion and a charged lepton/antilepton pair proceed via a flavour changing neutral current and therefore may only be induced beyond tree level in the Standard Model. This natural suppression makes these decays sensitive to the effects of potential New Physics. To discern such New Physics one must be able to control the errors on the Standard Model prediction of the decay amplitude. These particular decay channels however are dominated by a single photon exchange; this involves a sizeable long-distance hadronic contribution which represents the current major source of theoretical uncertainty. Here we outline our methodology for the computation of the long distance contributions to these rare decay amplitudes using lattice QCD, and present the numerical results of some exploratory studies using the Domain Wall Fermion ensembles of the RBC and UKQCD collaborations.

The 33rd International Symposium on Lattice Field Theory

14 - 18 July 2015

Kobe International Conference Center, Kobe, Japan

* Speaker. 


\section{Introduction}

The processes $K \rightarrow \pi \ell \bar{\ell}$ are interesting phenomenologically as they involve a flavour changing neutral current (FCNC). FCNCs are forbidden at tree level in the Standard Model (SM); the natural suppression of $K \rightarrow \pi \ell \bar{\ell}$ amplitudes makes them sensitive to potential New Physics. These decays may also be used to determine SM parameters such as $V_{t d}$ and $V_{t s}$, study CP violation and to test chiral perturbation theory (ChPT) descriptions of QCD at low energies [1].

The decays $K^{+} \rightarrow \pi^{+} \ell^{+} \ell^{-}$and $K_{S} \rightarrow \pi^{0} \ell^{+} \ell^{-}$are dominated by long distance effects induced by the one-photon exchange amplitude $[2,3]$. There is a significant contribution to this amplitude in the region where the photon emission and the $W$ exchange are separated by distances as large as $1 / \Lambda_{Q C D}$. Such long distance effects contain significant non-perturbative contributions, and so naturally we can use lattice QCD to evaluate them.

We aim to compute the Minkowski amplitude of the process $K \rightarrow \pi \gamma^{*}$, i.e.

$$
\mathscr{A}_{\mu}\left(q^{2}\right)=\int d^{4} x\left\langle\pi(\mathbf{p})\left|T\left[J_{\mu}(0) H_{W}(x)\right]\right| K(\mathbf{k})\right\rangle .
$$

Using electromagnetic gauge invariance this non-local matrix element can be written as

$$
\mathscr{A}_{\mu}\left(q^{2}\right) \equiv \frac{V(z)}{4 \pi^{2}}\left(q^{2}(k+p)_{\mu}-\left(m_{K}^{2}-m_{\pi}^{2}\right) q_{\mu}\right)
$$

where non-perturbative QCD effects are contained in the form factor $V(z), z=q^{2} / m_{K}^{2}$. This form factor has been parametrised in the context of chiral perturbation theory, in the form $[4,5]$

$$
V(z)=a+b z+V_{\pi \pi}(z),
$$

The component $V_{\pi \pi}(z)$ represents the contribution coming from $\gamma^{*} \rightarrow \pi \pi$ effects. The contribution of excited states is encapsulated in the polynomial term $a+b z$, the coefficients of which have previously been determined from experimental data [1]. One opportunity of lattice QCD is to test this relation by determining the constants $a$ and $b$ from simulation data.

This paper is organised as follows. In section 2 we discuss the implementation of this decay on the lattice. In section 3 we go on to discuss the analysis of the correlators computed on the lattice such that the matrix element of the decay may be extracted. We discuss the numerical results of our exploratory studies in section 4 . Finally in section 5 we make our conclusions.

\section{Lattice Methodology}

In order to extract the decay amplitude on the lattice we measure the 'unintegrated' 4pt correlator [3]

$$
\Gamma_{\mu}^{(4)}\left(t_{H}, t_{J}, \mathbf{k}, \mathbf{p}\right)=\sum_{\mathbf{x}, \mathbf{y}} e^{-i \mathbf{q} \cdot \mathbf{x}}\left\langle\phi_{\pi}\left(t_{\pi}, \mathbf{p}\right) T\left[J_{\mu}\left(t_{J}, \mathbf{x}\right) H_{W}\left(t_{H}, \mathbf{y}\right)\right] \phi_{K}^{\dagger}(0, \mathbf{k})\right\rangle
$$

where the operator $\phi_{P}(t, \mathbf{p})$ is the annihilation operator for a pseudoscalar meson $P$ with momentum $\mathbf{p}$ at a time $t$. The effective weak Hamiltonian relevant to the transition $s \rightarrow d \ell^{+} \ell^{-}$, renormalised at a scale $M_{W} \gg \mu>m_{c}$, is defined by [6]

$$
H_{W}=\frac{G_{F}}{\sqrt{2}} V_{u s}^{*} V_{u d}\left(\sum_{j=1}^{2} C_{j}\left(Q_{j}^{u}-Q_{j}^{c}\right)+\sum_{j=3}^{8} C_{j} Q_{j}+\mathscr{O}\left(\frac{V_{t s}^{*} V_{t d}}{V_{u s}^{*} V_{u d}}\right)\right) .
$$


In our implementation we consider only the operators $Q_{1}^{u, c}$ and $Q_{2}^{u, c}$; all others vanish at tree level and have much smaller Wilson coefficients than these two. The operators $Q_{1,2}^{q}$ are defined as [2,3]

$$
Q_{1}^{q}=\left(\bar{s}_{i} \gamma_{\mu}^{L} d_{i}\right)\left(\bar{q}_{j} \gamma^{L, \mu} q_{j}\right) \& Q_{2}^{q}=\left(\bar{s}_{i} \gamma_{\mu}^{L} d_{j}\right)\left(\bar{q}_{j} \gamma^{L, \mu} q_{i}\right)
$$

where $i, j$ are summed colour indices and $\gamma_{\mu}^{L}=\gamma_{\mu}\left(1-\gamma_{5}\right)$. To make contact with the continuum we first must non-perturbatively renormalise our lattice operators; we can then use perturbation theory to match with the Wilson coefficients for the $\overline{\mathrm{MS}}$ scheme, which are known at NLO [6]. The electromagnetic current $J_{\mu}$ is taken to be the standard flavour-diagonal current

$$
J_{\mu}=\frac{1}{3}\left(2 V_{\mu}^{u}-V_{\mu}^{d}-V_{\mu}^{s}+2 V_{\mu}^{c}\right)
$$

where $V_{\mu}^{q}$ is the conserved vector current for the flavour $q$. As this current is conserved it requires no renormalisation.

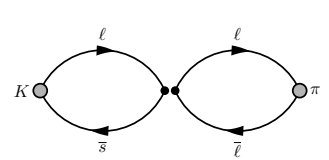

$W$

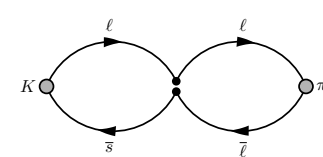

$C$

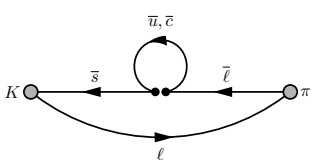

$S$

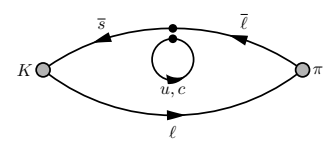

E

Figure 1: The four diagram topologies obtained after performing the Wick contractions for the $H_{W}$ operator.

In Fig. 1 we display the diagram topologies obtained by performing Wick contractions for just the $H_{W}$ operator. The current can be inserted on any of the quark propagators in each class; however there is also the possibility of the self-contraction of the current to produce a disconnected diagram, corresponding to a sea quark loop emitting a photon. The full list of diagrams can be found in Ref. [3]. When the current is inserted in the loop of the $S$ and $E$ diagrams, they appear quadratically divergent as the operators $J_{\mu}$ and $H_{W}$ approach each other. However, with gauge invariance and the GIM mechanism it can be shown explicitly $[2,3]$ that these diagrams introduce no new divergences.

\section{Analysis}

To recover the amplitude of the decay we consider the integrated 4pt correlator,

$$
I_{\mu}\left(T_{a}, T_{b}, \mathbf{k}, \mathbf{p}\right)=e^{-\left(E_{\pi}(\mathbf{p})-E_{K}(\mathbf{k})\right) t_{J}} \int_{t_{J}-T_{a}}^{t_{J}+T_{b}} d t_{H} \tilde{\Gamma}_{\mu}^{(4)}\left(t_{H}, t_{J}, \mathbf{k}, \mathbf{p}\right)
$$

in the limit $T_{A}, T_{B} \rightarrow \infty$, where

$$
\tilde{\Gamma}_{\mu}^{(4)}=\frac{\Gamma_{\mu}^{(4)}}{Z_{\pi K}}, Z_{\pi K}=\frac{Z_{\pi} Z_{K}^{\dagger}}{4 E_{\pi}(\mathbf{p}) E_{K}(\mathbf{k})} e^{-t_{\pi} E_{\pi}(\mathbf{p})} .
$$

The spectral decomposition for the integrated $4 \mathrm{pt}$ correlator is

$$
\begin{aligned}
I_{\mu}\left(T_{A}, T_{B}, \mathbf{k}, \mathbf{p}\right)= & -\sum_{n} \frac{1}{2 E_{n}} \frac{\left\langle\pi(\mathbf{p})\left|J_{\mu}\right| n, \mathbf{k}\right\rangle\left\langle n, \mathbf{k}\left|H_{W}\right| K(\mathbf{k})\right\rangle}{E_{K}(\mathbf{k})-E_{n}}\left(1-e^{\left(E_{K}(\mathbf{k})-E_{n}\right) T_{A}}\right) \\
& +\sum_{m} \frac{1}{2 E_{m}} \frac{\left\langle\pi(\mathbf{p})\left|H_{W}\right| m, \mathbf{p}\right\rangle\left\langle m, \mathbf{p}\left|J_{\mu}\right| K(\mathbf{k})\right\rangle}{E_{m}-E_{\pi}(\mathbf{p})}\left(1-e^{-\left(E_{m}-E_{\pi}(\mathbf{p})\right) T_{B}}\right) .
\end{aligned}
$$


The states $m$ must have the same quantum numbers of a kaon, and thus all possible states will have $E_{m}>E_{\pi}(\mathbf{p})$; this half of the integral thus converges as $T_{B} \rightarrow \infty$. However the states $n$ have the quantum numbers of a pion. For physical pion and kaon masses there are three permitted onshell intermediate states (namely one, two and three pion intermediate states), which will cause the integral to diverge with increasing $T_{A}$. These on-shell intermediate states do not contribute to the overall decay width and therefore must be removed in order to extract the relevant Minkowski amplitude,

$$
\mathscr{A}_{\mu}\left(q^{2}\right)=-i \lim _{T_{a}, T_{b} \rightarrow \infty} \tilde{I}_{\mu}\left(T_{A}, T_{B}, \mathbf{k}, \mathbf{p}\right),
$$

where $\tilde{I}_{\mu}$ indicates the integrated $4 \mathrm{pt}$ correlator after subtracting the divergent contributions.

\subsection{Single Pion Intermediate State}

Our exploratory simulations use an unphysically heavy pion mass of $\sim 420 \mathrm{MeV}$, and so we worry only about the single pion divergence; below we discuss the methods of its removal. A discussion of the $\pi \pi$ and $\pi \pi \pi$ divergences can be found in Ref. [3].

The first possibility is to reconstruct the analytical form of the divergence from Eq. (3.3). The divergent contribution is therefore

$$
D_{\pi}\left(T_{a}, \mathbf{k}, \mathbf{p}\right)=\frac{1}{2 E_{\pi}(\mathbf{k})} \frac{\mathscr{M}_{J, \pi}^{\mu}(\mathbf{q}) \mathscr{M}_{H}(\mathbf{k})}{E_{K}(\mathbf{k})-E_{\pi}(\mathbf{k})} e^{\left(E_{K}(\mathbf{k})-E_{\pi}(\mathbf{k})\right) T_{A}},
$$

where $\mathscr{M}_{\mu}^{J, P}(\mathbf{q})=\left\langle P(\mathbf{p})\left|J_{\mu}\right| P(\mathbf{k})\right\rangle$ and $\mathscr{M}_{H}(\mathbf{k})=\left\langle\pi(\mathbf{k})\left|H_{W}\right| K(\mathbf{k})\right\rangle$. The necessary matrix elements and energies can easily be recovered from fits to $2 \mathrm{pt}$ and $3 \mathrm{pt}$ correlators. In our exploratory studies the single pion state is the only divergent state, and so we could alternatively determine the contribution by fitting the $4 \mathrm{pt}$ function directly. We remark that for the $4 \mathrm{pt}$ case we do not fit the exponent of the $T_{a}$ dependence, which we can determine much more reliably from fits to $2 \mathrm{pt}$ correlators. We will refer to this divergence subtraction method as "method 1".

A second method ("method 2") of removing the single pion state is to employ a shift of the weak Hamiltonian by an unphysical scalar density, $\bar{s} d$ [7]. We choose a constant $c_{s}$ such that

$$
\left\langle\pi\left|H_{W}^{\prime}\right| K\right\rangle=\left\langle\pi\left|H_{W}\right| K\right\rangle-c_{s}\langle\pi|\bar{s} d| K\rangle=0 .
$$

By replacing $H_{W}$ by $H_{W}^{\prime}$ in Eq. (3.3) it is straightforward to see that the divergent single pion contribution vanishes. We can show that this shift leaves the total amplitude invariant using the chiral Ward identity

$$
i\left(m_{s}-m_{d}\right) \bar{s} d=\partial_{\mu} V_{\bar{s} d}^{\mu}
$$

\section{Numerical Results}

Our integration of the $4 \mathrm{pt}$ correlator requires us to simulate with a long enough time extent between the kaon source at $t_{K}$, the current insertion at $t_{J}$ and the pion sink at $t_{\pi}$. This is necessary to allow the integral to converge to extract the desired matrix element. To increase the time extent over which we can integrate the rare kaon $4 \mathrm{pt}$ correlator, we find that it is useful to consider two $4 \mathrm{pt}$ correlators with different values of $t_{J}$, requiring the computation of additional sequential propagators. By using a current insertion closer to the pion sink, we obtain a longer time extent over 
which to integrate in the region $\left[t_{J}-T_{A}, t_{J}\right]$. Likewise by inserting the current closer to the kaon source, we obtain a longer time extent to integrate over $\left[t_{J}, t_{J}+T_{B}\right]$. Hence we can obtain the two halves of the integral from these separate correlators and combine them appropriately to obtain the final result.

We ran a set of simulations with a kaon at $t_{K}=0$ with momentum $\mathbf{k}$ decaying into a pion at $t_{\pi}=34$ with momentum $\mathbf{p}$, with two separate current insertions as described above. We make use of Coulomb gauge-fixed wall sources for the pion and kaon to provide good overlap with the ground state. For the current insertion we make use of sequential propagators. The loops for the $S$ and $E$ (and disconnected) diagrams can be calculated using all-to-all propagators. However for our exploratory calculations we omit these diagrams to decrease the simulation time in order to develop our analysis techniques. Three different kinematics have been studied so far, $\mathbf{k} \rightarrow \mathbf{p}=(0,0,0) \rightarrow$ $(1,0,0),(0,0,0) \rightarrow(1,1,0)$ and $(1,0,0) \rightarrow(0,0,0)$ (momentum given as multiples of $2 \pi / L)$. All of our results were found using a sample of 256 configurations of a $24^{3} \times 64$ lattice with an inverse lattice spacing of $1 / a=1.73 \mathrm{GeV}$, employing domain wall fermions with Iwasaki gauge action, a pion mass of $\sim 420 \mathrm{MeV}$ and a kaon mass of $\sim 600 \mathrm{MeV}$ [8]. For the renormalisation of $H_{W}$ we may use the results of Ref. [9]; where a lattice of the same spacing, but smaller volume was used to perform the necessary NPR. We expect the results to hold for our lattice too as the renormalisation procedure depends upon the UV behaviour of the theory and thus is insensitive to finite volume effects.

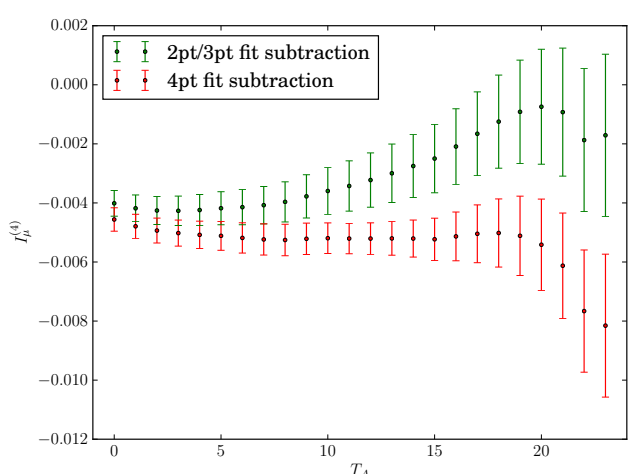

(a)

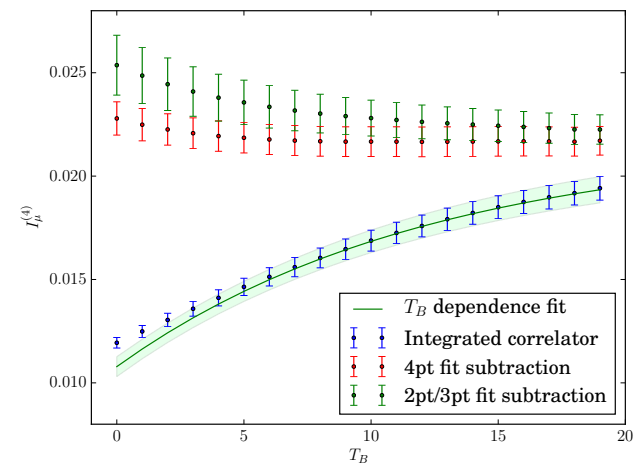

(b)

Figure 2: The dependence of the integrated 4pt correlator on the limits $(a) T_{A}$ (with $T_{B}=19$ fixed) and $(b)$ $T_{B}$ (with $T_{A}=4$ fixed). In $(a)$ the divergence has been removed using method 1 .

We display the dependence of the integrated 4pt correlator on the limits $T_{A}$ and $T_{B}$ in Fig. 2, removing the single pion divergence using method 1 . The fit of the $4 \mathrm{pt}$ function appears to remove the divergence more cleanly that by using the reconstruction from $2 \mathrm{pt} / 3 \mathrm{pt}$ fits. More investigation is necessary to understand the source of this discrepancy. We also observe that the $T_{B}$ dependence does not converge in the available time extent. This can be attributed to the fact that here the kaonpion mass difference is rather small, hence a small exponent for the decay. In practice it is thus necessary to remove this decay in a manner similar to the divergence.

We display the dependence of the integral of the $4 \mathrm{pt}$ function for the $K(0,0,0) \rightarrow \pi(1,0,0)$ kinematic in Fig. 3, where the divergence has been removed using method 2. We note the integral converges to a plateau within statistical errors for both sides of the integral. The parameter $c_{s}$ 


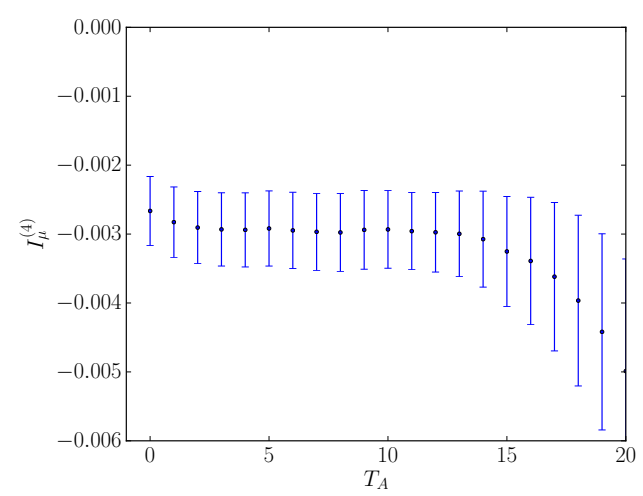

(a)

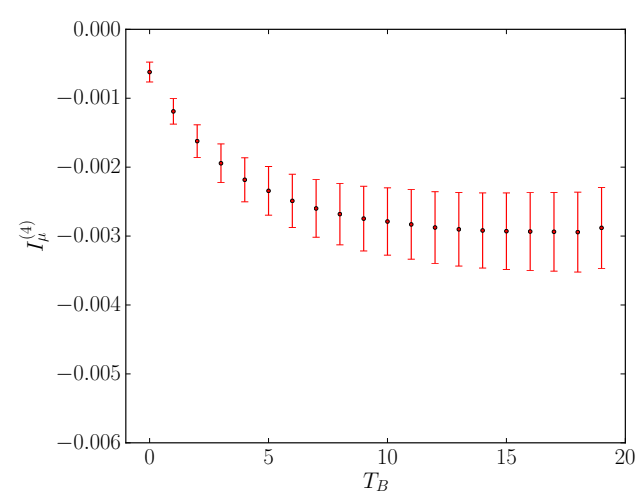

(b)

Figure 3: The dependence of the integrated 4pt correlator on the limits $(a) T_{A}$ (with $T_{B}=14$ fixed) and $(b)$ $T_{B}$ (with $T_{A}=7$ fixed), having removed the single pion divergence using method 2 .

\begin{tabular}{|c|c|c|}
\hline $\mathbf{k} \rightarrow \mathbf{p}$ & Method 1 (4pt fit) & Method 2 $(\bar{s} d$ shift $)$ \\
\hline$(0,0,0) \rightarrow(1,0,0)$ & $-0.0029(6)$ & $-0.0030(6)$ \\
\hline$(0,0,0) \rightarrow(1,1,0)$ & $-0.0049(23)$ & $-0.0024(27)$ \\
\hline$(1,0,0) \rightarrow(0,0,0)$ & $-0.0018(11)$ & $-0.0001(7)$ \\
\hline
\end{tabular}

Table 1: The $\mu=0$ component of the matrix element (in lattice units) found with each analysis method.

has a relatively weak momentum dependence, and so the $\bar{s} d$ shift reduces the amplitude of the exponentially decaying contribution of the single pion state.

In Table 1 we present a summary of the results for the two methods of extracting the matrix element from the integrated $4 \mathrm{pt}$ correlator. The two methods generally agree within statistical errors. We remark that we can fit the $4 \mathrm{pt}$ function with an $\bar{s} d$ insertion in a similar manner to the original in order to verify that after removing the divergence the integral of the correlator is within error of zero. For the $K(1,0,0) \rightarrow \pi(0,0,0)$ case we identify that the contribution of the integrated $\bar{s} d$ correlator is more than one sigma different from zero. More investigation is required to identify the source of any potential systematic effects in our analysis procedure.

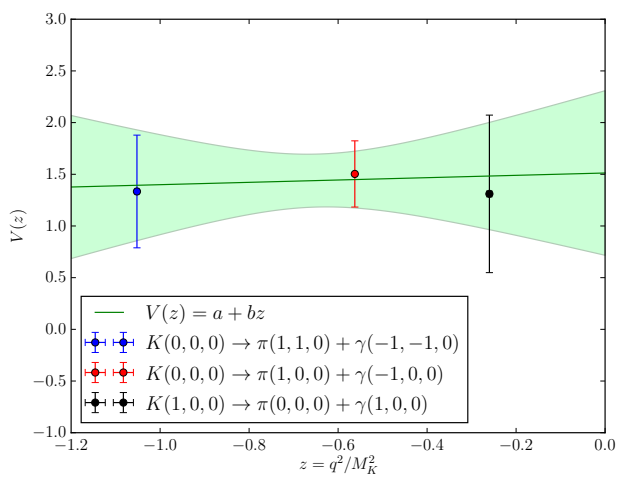

Figure 4: The dependence of the form factor on $q^{2}$ from our lattice simulations.

Finally we are able to use our results to determine the form factor for each kinematic. These 
results are shown in Fig. 4, where we have used the matrix element obtained by fitting the 4pt function directly. The fit ansatz is motivated by the ChPT prediction; we omit the $\pi \pi \rightarrow \gamma^{*}$ contribution here as it is negligible. At our current level of statistics the form factor does not appear to display any discernible dependence on $q^{2}$. We obtain the fit parameters $a=1.5(8)$ and $b=0.1(1.1)$. For contrast the experimentally determined parameters are $a=-0.578(16), b=-0.779(66)$ for $K^{+} \rightarrow \pi^{+} e^{+} e^{-}$and $a=-0.575(39), b=-0.813(145)$ for $K^{+} \rightarrow \pi^{+} \mu^{+} \mu^{-}$[1]. The comparison of central values is meaningless given our unphysical parameters, however we note that our errors are an order of magnitude greater than experiment. A significant gain in statistics would be needed before our errors become competitive.

\section{Conclusions}

Through our exploratory numerical simulations we have evaluated the different analysis techniques for extracting the long distance contributions to the decay $K^{+} \rightarrow \pi^{+} \ell^{+} \ell^{-}$using lattice QCD. At present it remains to extend our simulations to include the full set of diagrams on the current lattice. We subsequently aim to study the decay with more physical kaon/pion masses where it will be important to also consider $\pi \pi$ or even $\pi \pi \pi$ divergent contributions.

\section{Acknowledgements}

A.L is supported by an EPSRC Doctoral Training Centre grant (EP/G03690X/1). N.H.C and X.F are supported by US DOE grant \#DE-SC0011941. A.P and C.T.S are supported by UK STFC Grant ST/L000296/1. A.J acknowledges the European Research Council under the European Community's Seventh Framework Programme (FP7/2007-2013) ERC grant agreement No. 279757.

\section{References}

[1] V. Cirigliano, G. Ecker, H. Neufeld, A. Pich, and J. Portoles, Kaon Decays in the Standard Model, Rev. Mod. Phys. 84 (2012) 399, [arXiv:1107.6001].

[2] G. Isidori, G. Martinelli, and P. Turchetti, Rare kaon decays on the lattice, Phys. Lett. B633 (2006) 75-83, [hep-lat/0506026].

[3] RBC, UKQCD Collaboration, N. H. Christ, X. Feng, A. Portelli, and C. T. Sachrajda, Prospects for a lattice computation of rare kaon decay amplitudes: $K \rightarrow \pi \ell^{+} \ell^{-}$decays, Phys. Rev. D92 (2015) 094512, [arXiv:1507.03094].

[4] G. Ecker, A. Pich, and E. de Rafael, $K \rightarrow$ pi Lepton+ Lepton-Decays in the Effective Chiral Lagrangian of the Standard Model, Nucl. Phys. B291 (1987) 692.

[5] G. D'Ambrosio, G. Ecker, G. Isidori, and J. Portoles, The Decays $K \longrightarrow>$ pi $l+l$ - beyond leading order in the chiral expansion, JHEP 9808 (1998) 004, [hep-ph/ 9808289 ].

[6] G. Buchalla, A. J. Buras, and M. E. Lautenbacher, Weak decays beyond leading logarithms, Rev. Mod. Phys. 68 (1996) 1125-1144, [hep-ph/9512380].

[7] Z. Bai, N. Christ, T. Izubuchi, C. Sachrajda, A. Soni, et al., $K_{L}-K_{S}$ mass difference from lattice QCD, Phys. Rev. Lett. 113 (2014) 112003, [arXiv: 1406.0916$].$

[8] RBC, UKQCD Collaboration, Y. Aoki et al., Continuum Limit Physics from 2+1 Flavor Domain Wall QCD, Phys. Rev. D83 (2011) 074508, [arXiv: 1011.0892 ].

[9] RBC, UKQCD Collaboration, N. Christ, T. Izubuchi, C. Sachrajda, A. Soni, and J. Yu, Long distance contribution to the KL-KS mass difference, Phys. Rev. D88 (2013) 014508, [arXiv: 1212.5931]. 